\title{
ARTICLE
}

Multiple myeloma gammopathies

\section{Dynamic CD138 surface expression regulates switch between myeloma growth and dissemination}

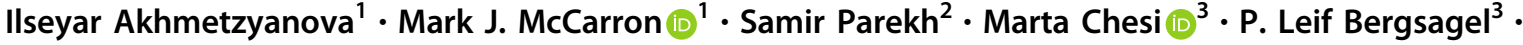 \\ David R. Fooksman ${ }^{1}$
}

Received: 25 January 2019 / Revised: 2 May 2019 / Accepted: 10 May 2019 / Published online: 22 August 2019

(c) The Author(s), under exclusive licence to Springer Nature Limited 2019

\begin{abstract}
The canonical plasma cell marker CD138 (syndecan-1) is highly expressed on the myeloma cell surface, but its functional role in vivo is unclear, as well as the ontogeny of CD138-high and CD138-negative (neg) myeloma cells. In this study we used an in vivo murine Vk*MYC myeloma model where CD138 is heterogeneously expressed depending on tumor size. We find that in comparison to CD138-neg myeloma cells, the CD138-high subset of myeloma cells is highly proliferative, less apoptotic, and enhanced IL-6R signaling, which is known to promote survival. In addition CD138-high myeloma engrafts better than its CD138-neg counterpart. In contrast, CD138-neg cells are more motile both in vitro and in vivo, and more readily disseminate and spread to other bones in vivo than CD138-high subset. Neutralizing CD138 rapidly triggers migration of myeloma cells in vivo and leads to intravasation, which results in increased dissemination to other bones. Both murine and human myeloma cells can rapidly recycle CD138 surface expression through endocytic trafficking, in response to serum levels. Blocking CD138 enhances myeloma sensitivity to bortezomib chemotherapy and significantly reduces tumor size compared to bortezomib treatment alone. Thus, our data show that CD138 surface expression dynamically regulates a switch between growth vs. dissemination for myeloma, in response to nutrient conditions.
\end{abstract}

\section{Introduction}

Patients with multiple myeloma, the second most common blood cancer in adults, have a median survival time of 5 years [1]. Myeloma is a malignancy in the plasma cell lineage, and shares many commonalities with normal plasma cells, including expression of the master transcriptional regulator, Blimp-1, expression of similar surface

These authors contributed equally: Ilseyar Akhmetzyanova, Mark J. McCarron

Supplementary information The online version of this article (https:// doi.org/10.1038/s41375-019-0519-4) contains supplementary material, which is available to authorized users.

David R. Fooksman

David.fooksman@einstein.yu.edu

1 Department of Pathology, Albert Einstein College of Medicine, Bronx, NY, USA

2 Department of Hematology-Oncology, Icahn School of Medicine at Mount Sinai, New York, NY, USA

3 Department of Medicine, Mayo Clinic, Phoenix, AZ, USA markers, and homing to the bone marrow (BM) [2]. Localization in the $\mathrm{BM}$ provides myeloma cells with critical survival cytokines, such as APRIL, IL-6, and SDF-1, which are important for survival and growth [3]. The BM stromal cells are also particularly critical for drug-resistance [4-6]. Thus, bone tropism combined with dissemination to multiple bones prevents any surgical interventions, and provides challenges for treatment [7].

One of the most abundant surface proteins on the surface of myeloma and plasma cells is syndecan-1 or CD138. Syndecan-1 is a large glycoprotein, carrying heparan sulfate moieties, expressed widely on BM hematopoietic cells, endothelial cells, and on some breast cancer cells. Among its many functions, CD138 has been implicated in wound healing [8], cell adhesion [9], endocytosis [10], and macropinocytosis [11], and may translocate to the nucleus [12]. Hundreds of proteins can bind to CD138, including extracellular matrix components and integrins for adhesion [13]. Although CD138 has no signaling function, it can bind and accumulate growth factors [14], cytokines [15], and chemokines [16] to enhance sensitivity and signaling indirectly. The ectodomain of CD138 can also be shed, which can affect its function and stability [17, 18]. Despite its high- 
expression level on myeloma cells, antibody targeted therapies against CD138 have failed as single agents for unknown reasons, but are currently being investigated in combination with other treatments or conjugated with various adjuvants or radio-isotopes [19-21].

We previously analyzed CD138 function on normal antibody secreting cells (ASCs) generated in vivo following immunization and found that CD138 was critical for antibody responses, by promoting survival of ASCs in a cellintrinsic manner [22]. This function was critically linked to APRIL and IL-6 receptor signaling, leading to higher expression of pro-survival factors, Bcl-2 and Mcll. We found that the increased sensitivity of CD138+ ASCs to exogenous IL-6 was dependent on heparan sulfate glycosylation on CD138, leading to more IL-6 receptor signaling via STAT3 phosphorylation. Indeed in contrast with wildtype hosts, we found that CD138+ ASCs had no survival advantage over CD138-deficient ASCs in IL-6 or APRIL deficient hosts, suggesting that cytokine binding is the primary function of CD138 on normal ASCs.

The general consensus is that CD138 expression is beneficial to tumor growth and progression [23], promoting survival and tumor growth in vitro and in subcutaneous models using xenografts [24]. CD138 has been shown to bind the survival factor APRIL [25], and has been shown to act as a co-receptor to bind growth factors, such as hepatocyte growth factor, epidermal growth factor, and IGF1 receptor, to promote cell survival and proliferation in the BM [26-28]. It has been shown that stable inhibition of CD138, by RNA-interference, reduces in vivo cell growth, enhances apoptosis and prevents dissemination in xenograft SCID models with limited BM involvement $[24,29]$. In addition, CD138 can be shed from the cells, during early stage of apoptosis in vitro [30] and increased serum level of shed, soluble CD138 is linked with poorer overall survival in myeloma [31], which may indirectly reflect increased tumor burden. However, patients with plasmacytomas lacking CD138 have worse prognosis [32], suggesting downregulation of CD138 may be linked to a clinically distinct stage of advanced disease.

There has been some debate to the nature of CD138-neg myeloma cells and their ontogeny. Matsui and colleagues have argued that the CD138-neg myeloma cells are dedifferentiated from the CD138+ population, with unique stem-like properties [33]. These findings have been disputed by findings from others [34, 35], which compared engraftment and growth of CD138+ and CD138- populations of various myeloma cell lines and human primary myeloma. In those studies they found no phenotypic or functional difference in CD138 subsets and the CD138 expression was not imprinted, however, the in vivo results relied on immunedeficient models or subcutaneous tumors, which may or may not be relevant to multiple myeloma that develops in the BM.
Therefore, with many conflicting roles identified in vitro and with regard to potential functions in vivo, the overall function of CD138 for myeloma physiology in vivo, particularly in an intact BM niche, remains unresolved. To address the functional role of CD138 in myeloma growth and dissemination in vivo in the BM of immunocompetent hosts, we made use of a murine $\mathrm{Vk} * \mathrm{MYC} \mathrm{GFP}^{+}$myeloma model of C57BL/6 (B6) origin [36, 37]. As tumor grows in the $\mathrm{BM}$, mice develop increased levels of monoclonal antibody (m-spike) and bone lytic lesions similar to patients with multiple myeloma. Using this model we find an unexpected and highly dynamic role for CD138 in vivo; at high levels, CD138 promotes myeloma growth, while at low or inhibited levels, it can promote dissemination. Importantly, these states are dynamic and reversible in response to serum, which could not be appreciated in a complete knockdown system and suggests a tightly regulated, conserved pathway.

\section{Methods}

\section{Mice, treatments, and myeloma administration}

C57BL/6 (B6) or congenic CD45.1+ (so called B6.SJL) mice were used as recipients for most experiments (Charles River). All mice were housed and bred in a specific pathogen-free animal facility at Albert Einstein College of Medicine. GFP-expressing Vk-MYC myeloma cells (Vk14451), obtained ex vivo from seeder recipients were stained for CD138 and FACS-purified based on GFP and CD138 expression. Equal numbers of cells were transferred in cohorts of mice, either by i.v. (1e4) or intratibial (1e3) injection. For IT-injected conditions, ex vivo BM cells were serum starved for $1 \mathrm{~h}$ to increase the pool of CD138-neg cells, then CD138-high and CD138-neg cells were sorted and injected. Serum-starved CD138-high cells behaved similarly to freshly isolated CD138-high cells indicating culturing was not a factor (data not shown). For comparison of i.v. injected subsets, donor cells were not serum starved but came from large tumors with abundant groups of CD138-high, intermediate, and negative cells.

In order to pool data from multiple independent experiments, normalization was used to account for variability in the initial engraftment after IT injection. To calculate dissemination index, myeloma total cell counts in contralateral tibial were divided by injected tibia total cell counts. For all experiments, experimental and control groups were littermates and cohoused in cages to ensure randomized groups. No subjective clinical scores were used therefore no blinding was applied in this study. Cohort sample sizes were estimated based on previous experiments with similar 
means and errors. All experiments were approved by the Institutional Animal Care and Use Committee.

\section{Myeloma functional assays}

Single-cell suspensions were prepared from spleen or BM by mechanical disruption, particulates were removed by filtration (70 micron BD filters) and red blood cells were lysed by treatment with ACK lysis (Lonza). For metastasis studies, BM cells from the primary injection site (primary tibia) and opposite tibia (contralateral tibia) were isolated and examined. For proliferation studies, mice were i.p. injected with $1 \mathrm{mg}$ EdU 1 day prior to sacrifice, and stained for CD138 and EdU using ClickIT kit (Invitrogen). Apoptosis and survival factors were stained as previously described [22]. Five micron transwells (corning) were used with total BM cells in RPMI media, with or without recombinant mouse SDF-1 ( $1 \mu \mathrm{g} / \mathrm{mL}$, R\&D Systems 460$\mathrm{SD})$ in the lower well. After $4 \mathrm{~h}$, cells were stained for CD138 and analyzed by flow cytometry using counting beads (Spherotech) to get total cell numbers. For pSTAT3 assays, ex vivo BM was serum starved for $1 \mathrm{~h}$ in RPMI media to reduce background levels and also promote CD138 downregulation. Exogenous IL-6 (R\&D Systems) was used at $10 \mathrm{ng} / \mathrm{mL}$. For CD138 recovery experiments, RPMI was supplemented with $10 \%$ of mouse plasma from BM or blood, FBS (Hyclone), or lipoprotein-depleted FBS (Kalen). Heparan sulfate levels were detected using by antibody staining (clone 10E4), as before [22]. For in vitro imaging, sorted GFP+ CD138+ myeloma cells were stained with SNARF-SE ( $5 \mu \mathrm{M}$ Invitrogen) and then plated with GFP + CD138 - myeloma cells on glass pre-coated with thin-coat of collagen (Pure-col) following manufacturer's protocol, and imaged at $37^{\circ} \mathrm{C}$ in media (HBSS media + HEPES buffer $+1 \%$ bovine serum albumin) using laser scanning microscope.

For anti-CD138 treatment experiments, mice were i.v. treated with $100 \mu \mathrm{g}$ purified anti-mouse CD138 antibody (clone 281-2, BioLegend) or matching isotype (rat IgG2a, BioLegend). For imaging experiments, mice were treated once acutely and imaged for $2-17 \mathrm{~h}$ post treatment. For intravasation myeloma measurements by flow cytometry, mice were bled prior to being treated twice, $(100 \mu \mathrm{g}$ each time, for 2 days) and analyzed $6 \mathrm{~h}$ after second dose, by bleeding. Counts of myeloma cells were normalized to $50 \mu \mathrm{l}$ of whole blood, using counting beads. For tumor burden with chronic anti-CD138 therapy, mice were treated once per week for 4 weeks and then analyzed a week later. For bortezomib treatments, mice received $0.5 \mathrm{mg} / \mathrm{kg}$ (assuming $20 \mathrm{~g}$ mouse) dissolved in 20\% DMSO in phosphatebuffered saline, i.p on days $2,5,8$, and 11 , following a previous design [37], with anti-CD138 or isotype treatment on days 0 and 7.

\section{Human myeloma}

Freshly collected human myeloma was obtained from BM aspirates from consenting patients and following an approved IRB. BM mononuclear cells were purified by Histopaque gradient, and stained using similar protocols as for mouse myeloma, but using antibodies specific for human myeloma. CD138 (clone B-A38) from Bio-Rad Laboratories was used. To identify myeloma cells, we used CD56+, CD20-, CD19-, CD138-high gating strategy, which revealed $\mathrm{CD} 138+$ and $\mathrm{CD} 138$-subsets, which were also sensitive to serum starvation.

\section{Intravital imaging and analysis}

Tiled BM Images were collected using Two-Photon Olympus Microscope (FVE-1200). For time-lapse movies, xyzt datasets were collected and analyzed for migration using semi-automated tracking on Volocity 6.3 (Improvision) and Imaris (Bitplane). Tracks were accumulated from multiple regions, and representative of three or more imaging sessions from independent experiments. Movies were annotated using Adobe After Effects.

\section{Statistical analysis}

Mean value and standard error of the mean were calculated. Unpaired, two-tailed Student $t$ tests or Mann-Whitney $t$ tests were used where appropriate to calculate significance between the two groups.

\section{Results}

\section{Decreased CD138 expression and increased cell motility correlates with tumor burden}

To study myeloma growth and image cells in vivo independently of phenotypic markers such as CD138, we used a transplantable Vk*MYC myeloma clone (Vk14451) that stably expresses GFP [38]. Intravenous transfer of $\mathrm{GFP}^{+}$cells led to development of clonal foci or clusters of myeloma in the BM (Fig. 1a) disseminated throughout the body. Within these clusters, individual myeloma cells were not attached to one another, as is the case with solid tumors (Supplemental Movie 1), and were sessile and not migrating based on time-lapse intravital two-photon imaging in the tibia (Fig. 1a, Supplemental Movie 1). However, as GFP+ myeloma cell clusters grew, they eventually dominated the BM parenchyma at advanced stages of disease (Fig. 1a), cells were more dynamic and motile by imaging (Supplemental Movie 1). To track individual cell motility within 
A

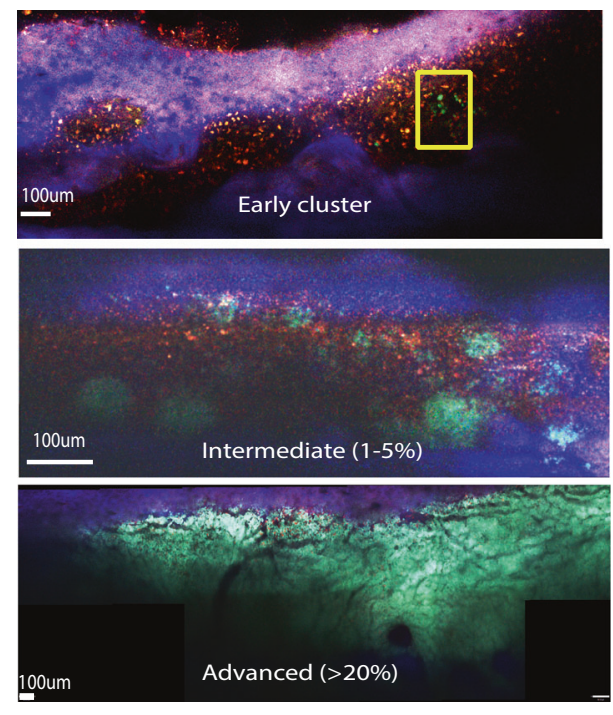

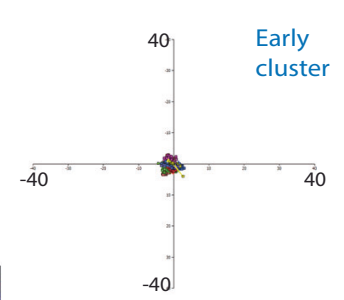
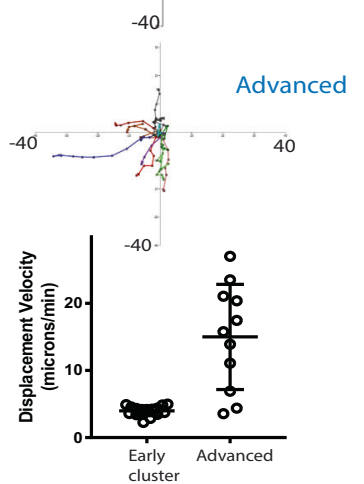

B
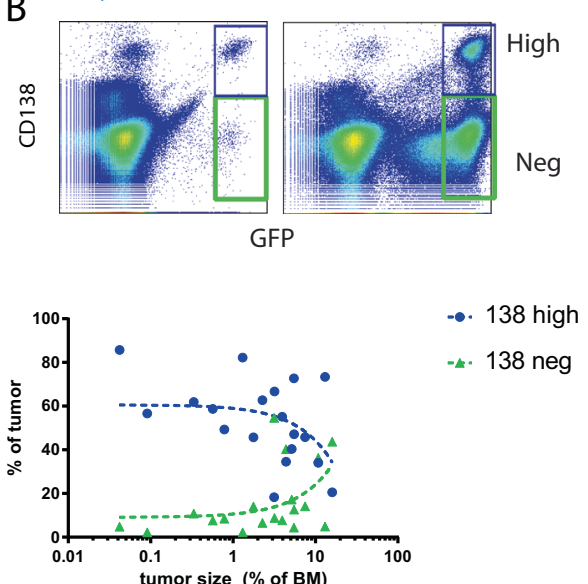

C

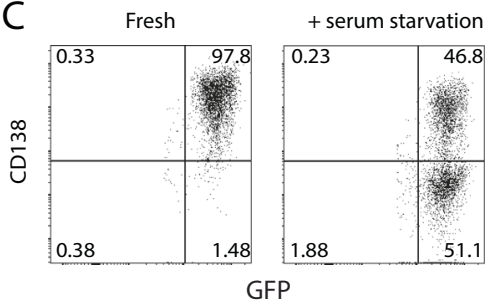

Fig. 1 CD138 expression and cell motility changes with tumor burden. a Intravital two-photon imaging of GFP+ (green) Vk*myc myeloma cells in the tibia at various tumor burdens (early, intermediate, and advanced stages) with analysis of individual cell track trajectories of total GFP + myeloma cells in the BM, arrested at early stage cluster (yellow box in (a)) and representative migrating cells taken from advanced stage ( $>20 \%$ tumor burden) using mixed tumor of GFP +

dense myeloma clusters at advanced stages, we engrafted a mixture of GFP+ myeloma cells with bortezomibresistant GFP - myeloma cells (Vk12598 clone) [39] and found some highly motile GFP + myeloma cells with displacement rates greater than $10 \mathrm{microns} / \mathrm{min}$ (Fig. 1a) and we could observe intravasation into the sinusoids (data not shown). Phenotypic analysis revealed that myeloma cells were predominantly CD138-high at early stages of disease, whereas when the tumor reached a frequency of $10 \%$ or higher of total BM cells, the fraction of CD138-neg cells increased greatly (Fig. 1b), suggesting CD138 expression may control or report on disease progression in vivo. We hypothesized that changes in CD138 expression may be related to increased tumor density leading to consumption and reduced levels of survival factors or nutrients in the BM microenvironment. To test this, we cultured freshly isolated tumor bearing BM cells in media lacking serum for $1 \mathrm{~h}$ and found a substantial drop in CD138 surface expression on myeloma on live cells, suggesting a highly regulated and dynamic response to extrinsic cues (Fig. 1c), which we investigate in further detail. and GFP-clones. b Representative dot plots of CD138 expression on myeloma cells (gated in black) at low and high tumor burden and overall trends in CD138-high and negative subsets plotted over various tumor sizes. c Comparison of CD138 expression on GFP+ myeloma cells either freshly isolated from BM, or after $1 \mathrm{~h}$ serum starvation (representative plot)

\section{CD138 expression promotes cell proliferation, survival, IL-6 signaling, and BM engraftment}

Based on variegated CD138 surface expression, GFP+ myeloma cells were subdivided into two groups, CD138high and CD138-neg (as in Fig. 1b), then analyzed for differences in in vivo function and physiology (Fig. 2a). CD138-high myeloma cells had higher proliferation than the CD138-neg subset, based on EdU incorporation in vivo or by ex vivo Ki-67 staining. Analysis of apoptosis following serum starvation [22], revealed that the CD138-neg population was more pro-apoptotic, both by Annexin $\mathrm{V}+$ or activated Caspase-3 staining, which correlated with decreased overall expression of pro-survival factors Bcl2, and Mcl1 compared to CD138-high populations in live cells. We previously showed that CD138 protected newly generated antibody secreting cells from cell death by promoting IL-6 and APRIL receptor signaling that resulted in greater expression of the downstream, respective, prosurvival proteins Bcl2 and Mcl1 [22]. Since the CD138high myeloma subset was less apoptotic than the CD138neg subset, we tested if cytokine signaling was the 

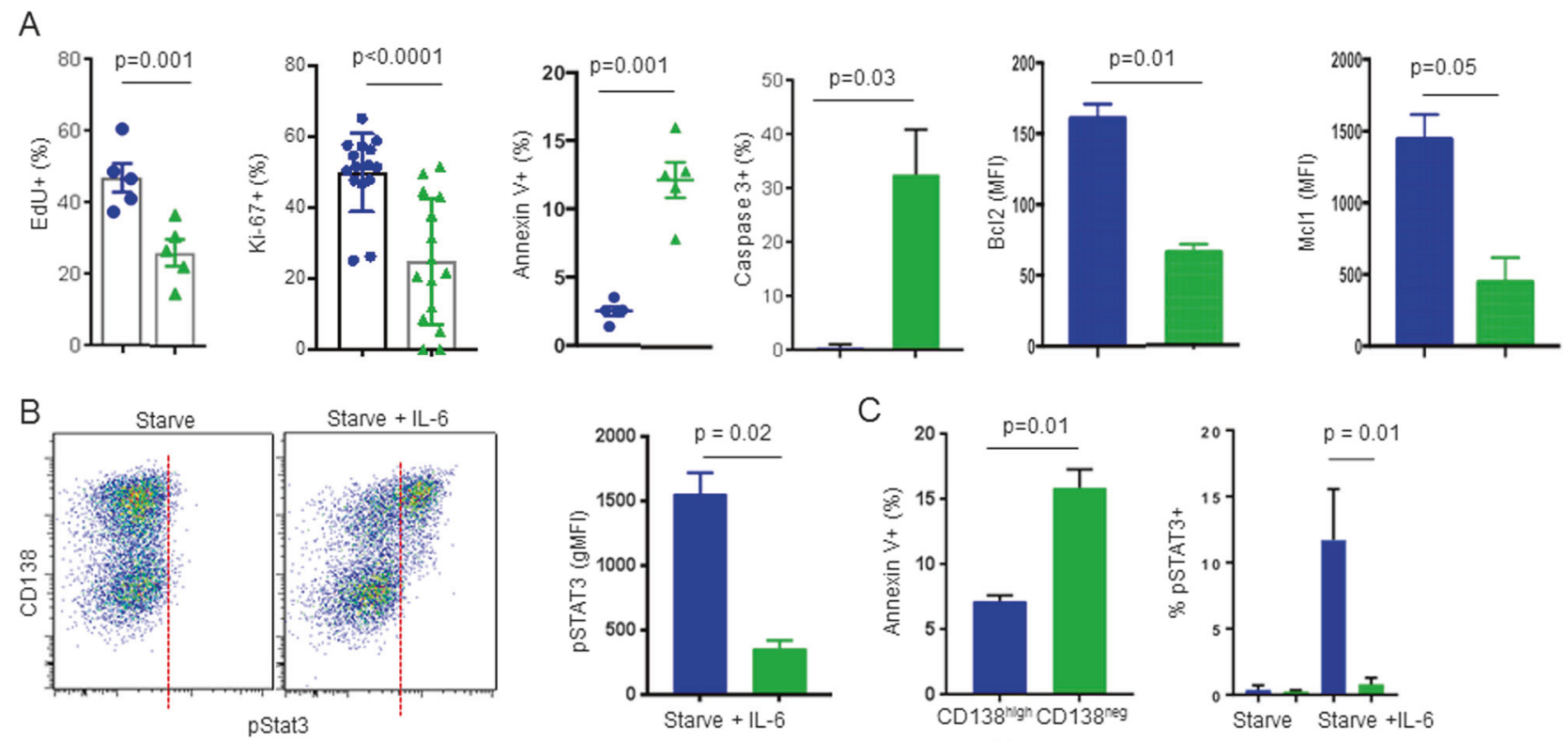

C
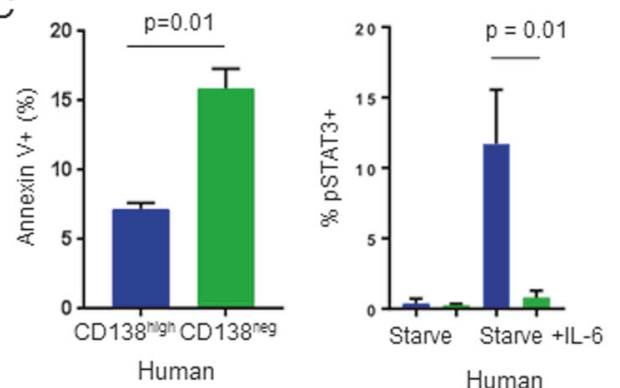

Fig. 2 CD138 expression correlates with cell survival, proliferation, and IL-6 receptor signaling. a Comparison of CD138-high (blue), and negative (or neg in green) myeloma GFP + subsets for proliferation, apoptosis, and survival factors from ex vivo fresh tumor, pre-gated on live cells. $n$ is shown on each graph. b Freshly isolated myeloma was treated with or without exogenous IL-6 and IL-6 receptor signaling assessed by pSTAT3 intracellular staining on CD138 subsets for murine cells $(n=3)$. c Comparison of CD138-high and CD138-neg human myeloma (CD19- CD20- CD56+ CD138-high) cells by Annexin V and pSTAT3 $(n=3)$. All experiments were independently repeated two or more times. Mann-Whitney $t$ tests were used. Errors reflect SD mechanism. Ex vivo stimulation of serum-starved myeloma cells with exogenous IL-6 led to IL-6 receptor activation, as assessed by phosphorylation of downstream transcription factor, STAT3 (pSTAT3). Activation of STAT3 was elevated in the CD138-high myeloma fraction only (Fig. 2b) indicating CD138 promotes IL-6 receptor sensitivity.

To extend these results, we analyzed myeloma taken from BM aspirates (gated based on myeloma surface markers Fig. S1). Human CD138-neg myeloma cells from patient BM aspirates gated on myeloma markers were more apoptotic compared to the CD138-high subset (Fig. 2c). IL6 treatment of human myeloma also induced pSTAT3 expression in a CD138 dependent manner as we saw in murine model.

To assess the role of CD138 in BM engraftment, equal numbers of FACS-purified CD138-high, CD138intemediate or CD138-neg cells were i.v. transferred into WT recipient mice that were sublethally irradiated to increase engraftment efficiency (Fig. 3a). At 8 weeks, mice receiving CD138-high injected donor cells developed larger tumors than mice that received the CD138-neg subset, based on higher M-spike, and myeloma cell counts and frequency in the BM (Fig. 3b). Interestingly, all recipient mice developed tumors with similar variegated CD138 expression pattern, irrespective of which donor CD138 subset was used (Fig. 3c), suggesting that the CD138-neg subset was not a precursor or stem-like population in this model. Since mice injected with CD138intermediate cells were not significantly different than CD138-high cells in terms of tumor growth suggesting overlap between the two groups and we focused on differences between CD138-high and negative subsets.

\section{CD138-neg myeloma cells are more motile and promote dissemination in vivo}

We noted that increased cell motility in larger tumors correlated with decreased CD138 expression (Fig. 1). Thus, motility was analyzed using a transwell assay. Using unpurified BM as input cells, which contained a mixture of 50\% CD138-high myeloma cells and 50\% CD138-neg myeloma cells, the majority $(\sim 90 \%)$ of myeloma cells that transmigrated were CD138-neg (Fig. 4a) in response to SDF-1. Using sorted CD138-high and CD138-neg myeloma cells tested separately, we also found that CD138-neg myeloma cells were more migratory by transwell assay (Fig. 4a). FACS-sorted and dye-labeled CD138-high and CD138-neg subsets were plated on collagen substrates to assess in vitro cell migration. Although we did not see any migration, we found that CD138-high cells adhered and clustered together rapidly (Fig. 4b), which would prevent transmigration in vitro.

We next examined if CD138 expression controlled BM retention and myeloma dissemination in vivo. To do so, we 
Fig. 3 CD138 expression promotes tumor engraftment and is dynamic in vivo.

a Experimental design: FACSpurified populations of CD138 subsets (color coded) were i.v. transferred into sublethally irradiated (300Rad) WT recipients and analyzed 8 weeks later. b Total tumor burden was assessed by serum M-spike anti-IgG2b ELISA, and flow cytometry in the BM shown (frequency and in total cell counts). c Analysis of CD138 expression on myeloma from cohorts of mice show no difference in distribution or expression. Data are representative of two independent experiments $(n=3$ mice per group per experiment). Mann-Whitney $t$ tests were used. Errors reflect SD
A

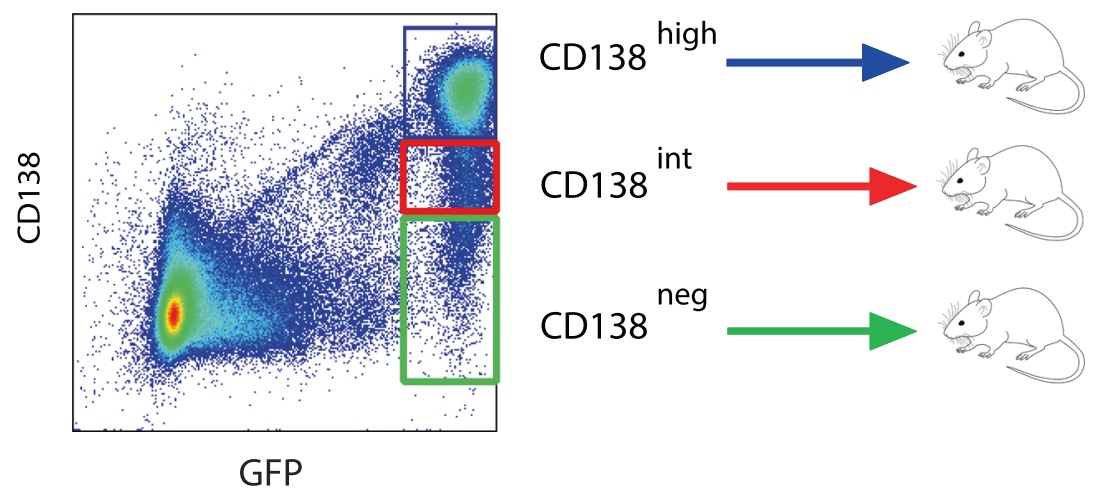

B
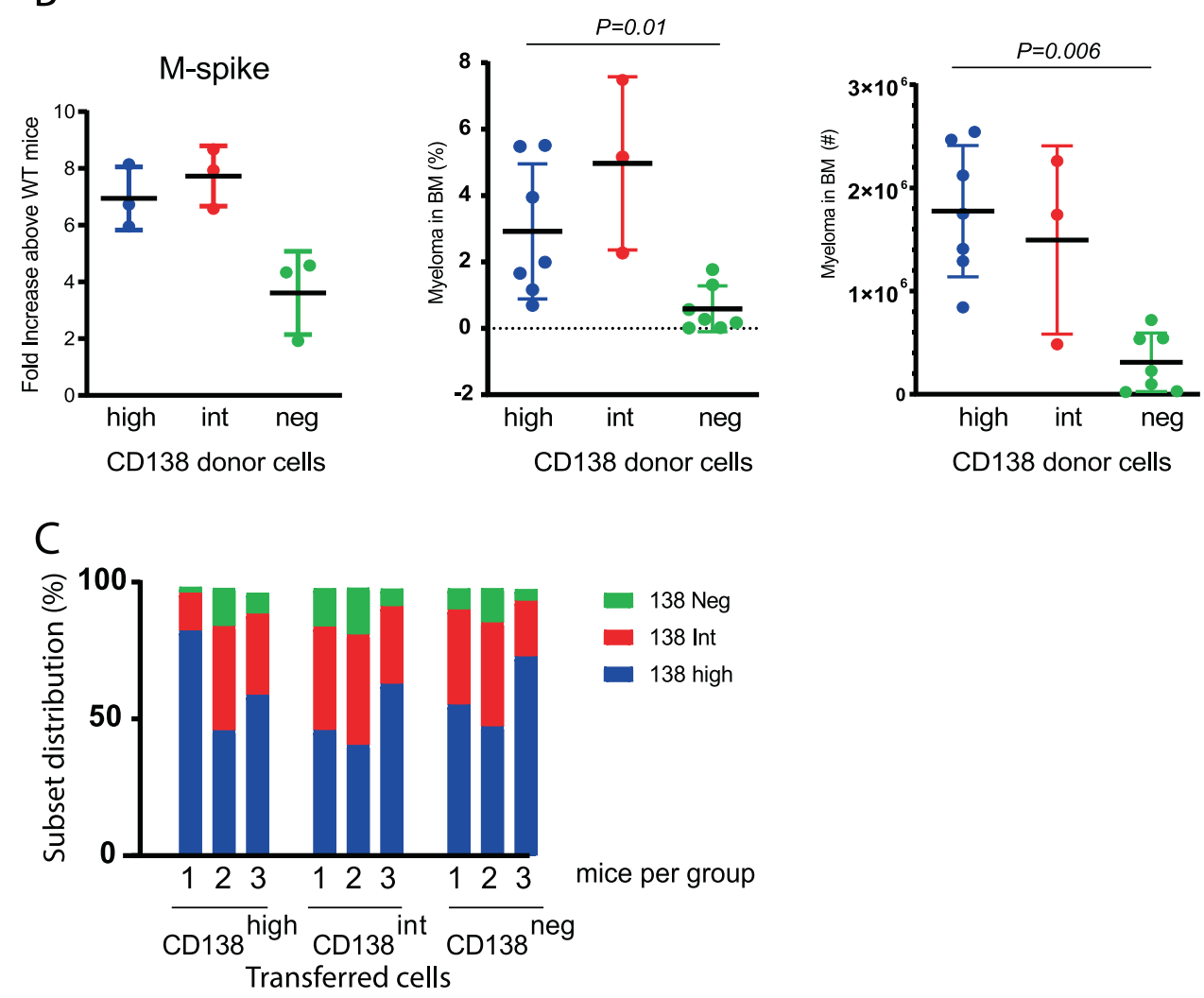

established a new transfer model by injecting myeloma cells into the left tibial BM to establish a single, primary myeloma site in the body. Following intratibial (IT) injection, tumor growth, and spreading was monitored every 2 weeks. Up to 4 weeks post injection, myeloma cells were only detectable in the injected (left) tibia, but by 6 weeks, spreading had occurred to other bones, and spleen, suggesting myeloma cells had spread from the primary site in the injected tibia after 4 weeks (Fig. S2). FACS-purified CD138-high or CD138-neg cells taken from serum-starved tumors were IT injected into recipients, to assess local growth and dissemination. CD138-high cells developed larger tumors in the injected bone compared to CD138-neg myeloma cells (Fig. 4c), in line with results from i.v. transfer of sorted CD138-high and CD138-neg populations taken from large tumors without serum starvation (Fig. 3a). However, CD138-neg cells showed an increased rate of dissemination and spread to the contralateral tibia (Fig. 4d), suggesting that CD138 downregulation mediates myeloma release and migration to distal sites. To validate these results, mice were IT-injected with a second $\mathrm{Vk} *$ myc clone (Vk12598), and found CD138-high cells grew larger tumors in the injected bone (Fig. 4c), while CD138-neg cells disseminated more efficiently to the contralateral tibia (Fig. 4d). In these IT-injected experiments, surface expression of CD138 in the engrafted tumors at 5-6 weeks 

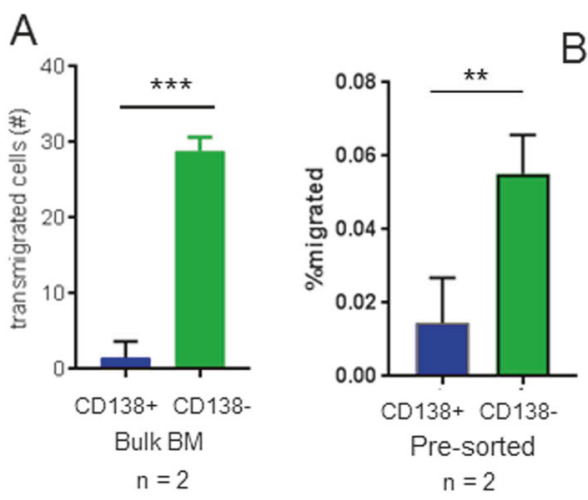

B
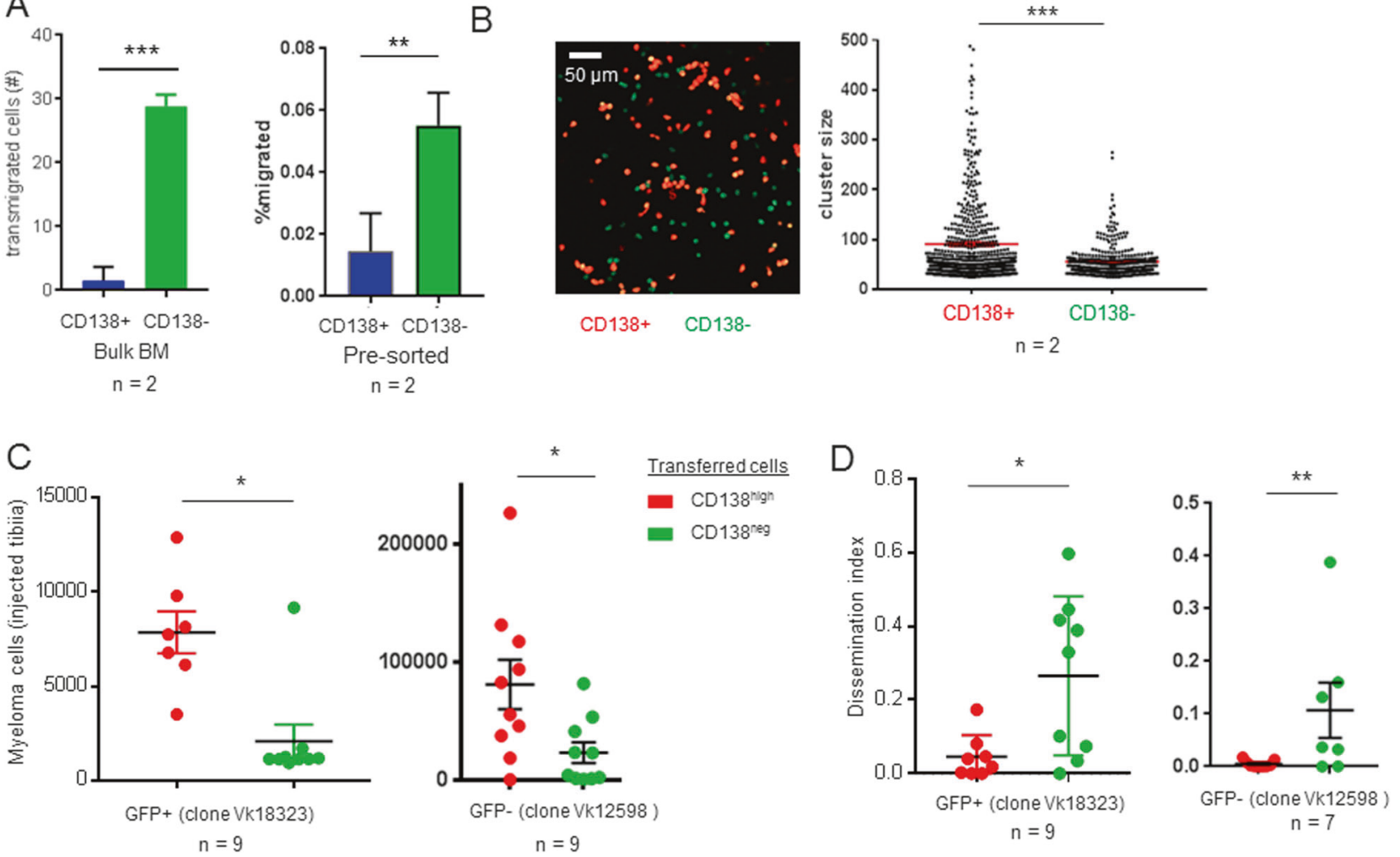

Fig. 4 CD138-neg myeloma cells are more motile in vitro and disseminate better in vivo. a Myeloma cell motility assessed by transwell assay. Percent frequency of CD138+ and CD138- myeloma cells in lower chamber using bulk tumor or FACS sorted and separately tested CD138+ and CD138 - subsets $(n=2)$. b Sorted and labeled CD138+ (red) and CD138 - (green) fractions were imaged on collagen coated glass, and cluster sizes were measured by image analysis software. c Analysis of tumor size in the injected (left) tibia 5 weeks after IT

injection of FACS-purified CD138 subsets using GFP+ (Vk14451 clone) or GFP- (Vk12598 clone) from ex vivo serum-starved tumors. Analysis of dissemination index (ratio of tumor burden in contralateral tibia over injected tibia) using both clones. Data in $\mathbf{d}-\mathbf{e}$ are pooled from two experiments for GFP + clone and one GFP - experiment shown that is representative of the second one as well. Means were analyzed by Mann-Whitney $t$ test. Errors reflect SEM in (a), and SD in (e)

was the same regardless of what cells were injected (data not shown), similar to what was found in the i.v injection model without starvation (Fig. 3c).

\section{Anti-CD138 rapidly mobilizes myeloma cells in the $\mathrm{BM}$ and promotes dissemination}

Although CD138 expression inversely correlated with migration, potentially CD138 expression or function were not directly responsible for this phenomenon. To examine this directly, mice with IT-injected tumors were treated with anti-CD138. Intravital imaging revealed increases in cell motility and intravasation as early as 2-h post treatment (Supplemental Movie 2, Fig. 5a). Although cells were more motile within tumor clusters, the overall tumor cluster did not expand in the field, while overall tumor volume was reduced tumor volume 1 day later (Fig. 5b). By time-lapse imaging, we could observe myeloma apoptosis occurring frequently in vivo following treatment, by the production of cellular debris, and also increased phagocytosis by auto-

fluorescent macrophages (Supplemental Movie 3, Fig. 5c). We confirmed increased mobilization into the blood by flow cytometry 1 day after anti-CD138 treatment (Fig. 5d), and myeloma cells had reduced CD138 surface levels by ex vivo staining, suggesting anti-CD138 was targeting myeloma directly (data not shown). Since CD138 is widely expressed in the body, we tested if myeloma-expressed CD138 was required for BM retention. CD138-competent myeloma cells were IT injected into CD138-deficient mice [40] and allowed tumors to develop. Following anti-CD138 treatment, we could see myeloma cells increased myeloma cell motility (Fig. 5a) and increased intravasation (Fig. 5d), confirming that anti-CD138 treatment was acting on myeloma cells directly to trigger dissemination.

To see the functional consequences of anti-CD138 therapy, we treated cohorts of tumor bearing mice weekly with anti-CD138 or isotype for 4 weeks beginning at tumor injection in the tibia. Tumor size was significantly smaller in the injected bone in anti-CD138 treated mice, however, dissemination and spreading to the contralateral tibia was 
A

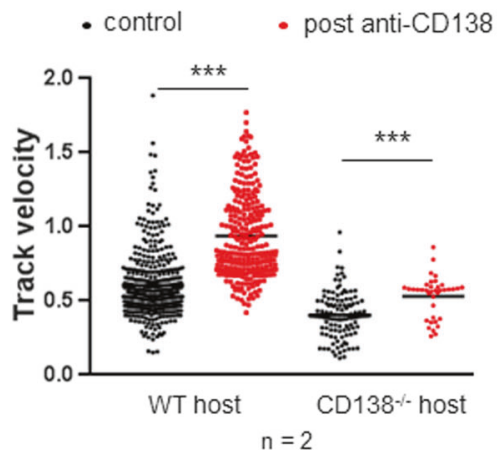

B

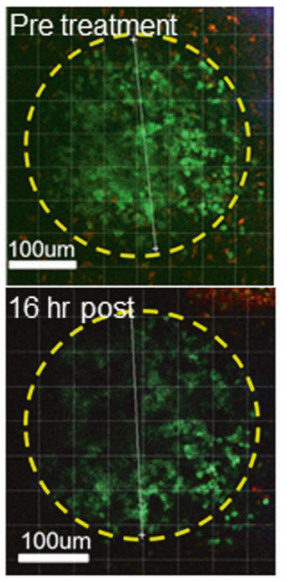

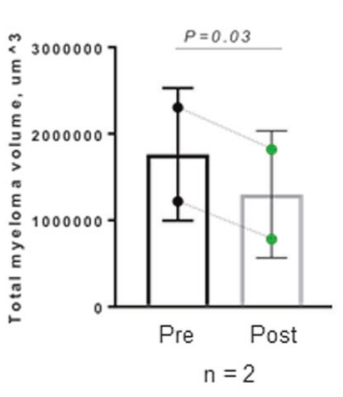

C

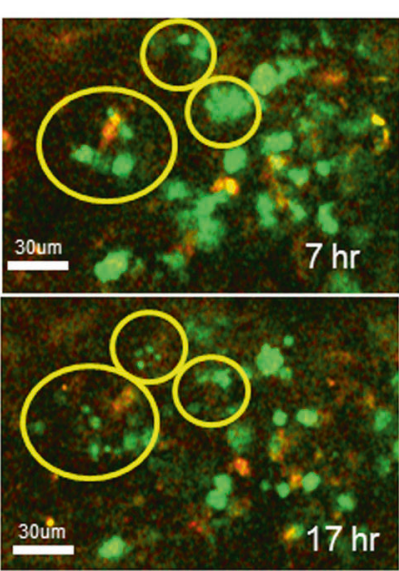

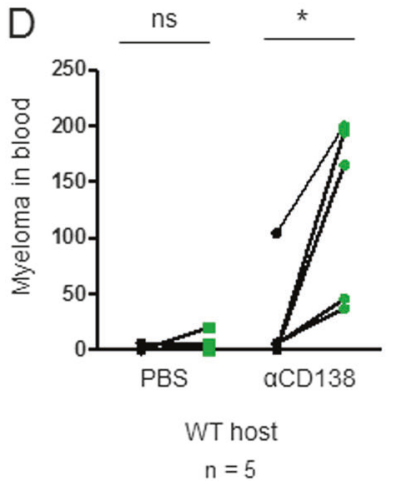

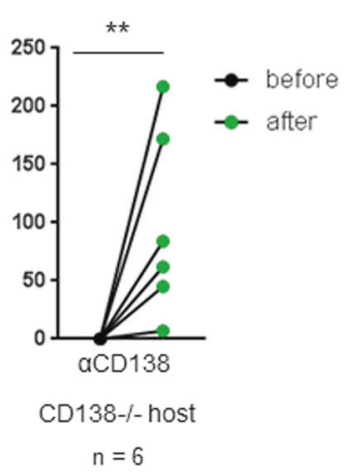

Fig. 5 Anti-CD138 rapidly mobilizes myeloma cells in the BM and promotes dissemination. a WT or CD138-/- recipient mice ITinjected myeloma, were imaged in the tibia, prior to (or untreated) with anti-CD138 (iv) or following treatment (2-6h post) by time-lapse imaging. Myeloma cell motility was measured and pooled from two mice per group. b Representative images and comparisons of tumor cluster volume (by image analysis of GFP intensity) pre and $17 \mathrm{~h}$ post

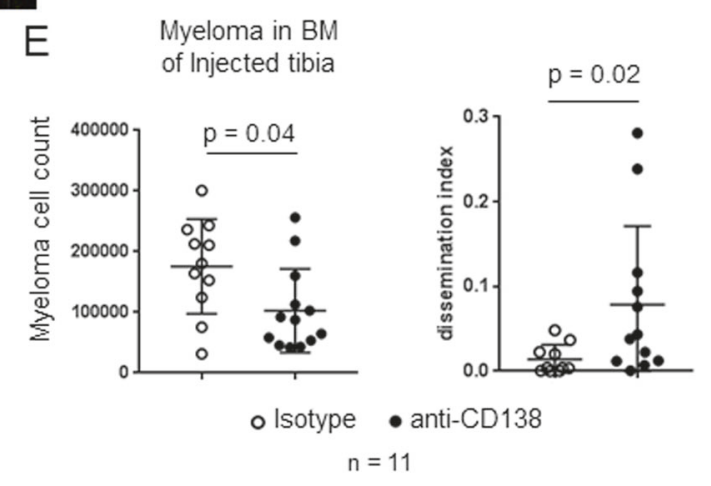

anti-CD138 treatment. c Images from time-lapse movies following treatment with circles highlighting apoptotic bodies. d Analysis of intravasation myeloma in blood prior to and following anti-CD138 treatment, PBS treatment in WT mice or in CD138-/- hosts. e Analysis of tumor burden in injected bone and dissemination index following chronic anti-CD138 or control antibody treatment for 4 weeks. $n=11$. Errors reflect SD significantly increased (Fig. 5e). Taken together, CD138 is directly responsible for the controlling myeloma retention and blocking CD138 is sufficient to trigger mobilization from the BM.

\section{CD138 surface expression is highly dynamic and sensitive to serum factors}

We next examined the dynamics of CD138 surface expression on myeloma cells, whose downregulation was detectable in vivo and could be rapidly triggered and modeled ex vivo by serum starvation (Fig. 1c). It was been reported that CD138 can be cleaved and shed [41], alternatively, rapid surface downregulation after serum starvation could also be due to internalization. To resolve this, cells were pre-stained with a mix of two monoclonal antiCD138 (clone 281.2) antibody conjugated protein fluorophore, phycoerthrin (PE) or polymer-based fluorophore Brilliant Violet 510 (BV510). We hypothesized that if the
CD138 ectodomain was shed, we would see a proportional decrease in PE and BV510 fluorescence on cells after serum starvation. In contrast, if internalization was at play, we would see a reduction in PE fluorescence, which is sensitive to $\mathrm{pH}$ and proteolysis, whereas BV510 polymers would be unaffected by internalization. Indeed flow cytometric analysis revealed a 75\% decrease in CD138-PE cell fluorescence after serum starvation, but only a $7 \%$ decrease in CD138-BV510 intensity, consistent with CD138 endocytic trafficking into an acidic or degradative compartment (Fig. 6a).

Since CD138-neg myeloma cells can regain CD138 surface expression in vivo (Fig. 3d), we checked if serum alone could restore CD138 surface expression after ex vivo serum starvation. Indeed, re-culturing cells in media with $10 \%$ FBS for $2 \mathrm{~h}$ restored CD138 surface expression (Fig. 6b). Based on this result, we tested the idea that CD138-neg cells exiting the BM could restore CD138 expression in the blood, thereby promoting extravasation at 
A
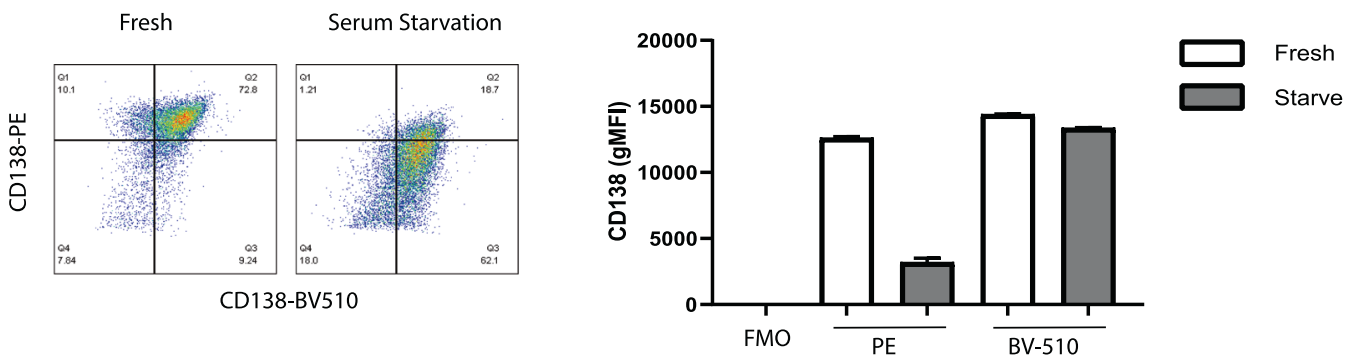

B
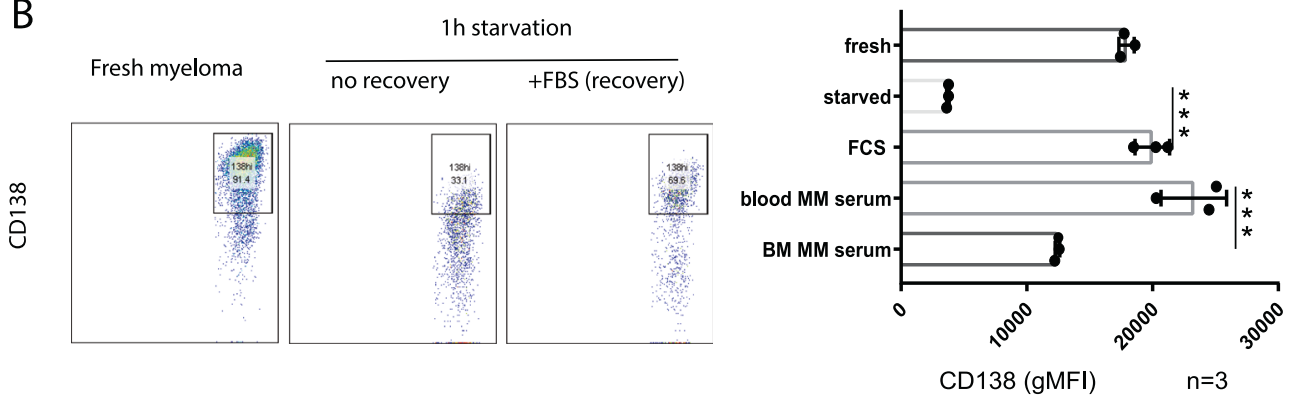

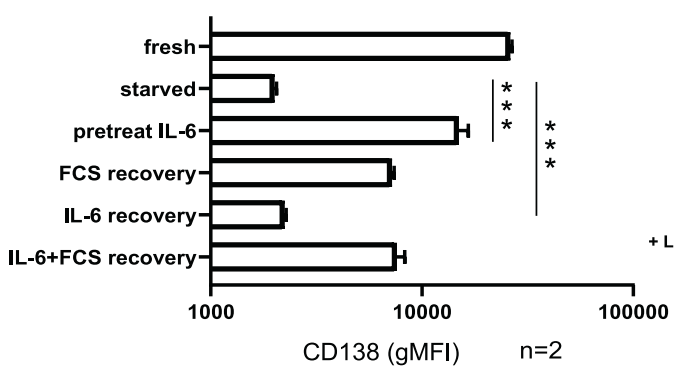

Fig. 6 CD138 surface expression is reversibly controlled by lipoproteins in serum. Freshly isolated myeloma cells were co-stained with PE anti-CD138 and BV510 anti-CD138, then serum starved $1 \mathrm{~h}$ and analyzed by flow cytometry. a Representative dot plots comparing PE and BV510 fluorescence before and after starvation, quantifying fluorescence intensity (geometric mean) for both fluorophores with

a new site. Treatment with mouse blood plasma from naïve or tumor bearing mice were equally capable of restoring CD138 expression. However, BM plasma taken from mice with high tumor burden was unable to fully restore CD138 expression, suggesting that the BM supernatant was depleted of these serum factors. Pre-treating CD138-high cells with IL-6 prevented starvation-induced CD138 downregulation, but IL-6 was not sufficient to restore CD138 surface levels following starvation. Lipoprotein-depleted FBS was unable to recover CD138 levels following starvation, nor was liproprotein-rich fractions (data not shown) suggesting multiple factors were required for recovery. Similar results were obtained using human myeloma from multiple myeloma patients (Fig. 6c), however, recovery was weaker using bovine serum, as compared to human serum from healthy adult bone, suggesting species specificity for these serum factors. Taken together, we find that CD138 expression is

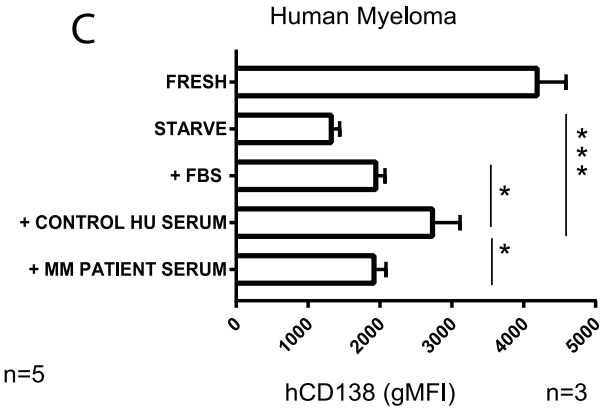

FMO control, representative of two independent experiments. b Serum-starved myeloma recovers CD138 expression following reculturing for $2 \mathrm{~h}$ with $10 \%$ FBS containing media, or other recovery conditions. c Human CD138 re-expression following starvation and serum recovery. Replicates pooled from independent experiments and analyzed by $t$ tests. ${ }^{*} p<0.05,{ }^{* * *} p<0.001$

highly sensitive to serum factors and can rapidly respond to both increases and decreases in serum.

\section{Anti-CD138 therapy enhances myeloma sensitivity to bortezomib chemotherapy}

Single agent anti-CD138 treatments have been effective in some preclinical models but not effective in human clinical trials, possibly due to myeloma mobilization (Fig. 5). Since BM stroma has been shown to block chemotherapy [6], and anti-CD138 mobilized myeloma cells into circulation, we assessed if anti-CD138 could enhance myeloma sensitivity to bortezomib treatment in vivo. We treated tumor bearing mice with bortezomib $(0.5 \mathrm{mg} / \mathrm{kg})$ with isotype control or antiCD138 as described (Fig. 7a). Combining bortezomib with anti-CD138 had a synergizing effect, reducing tumor size by more than ten-fold above single agent (Fig. 7b, Fig. 4d, e). 
Fig. 7 Anti-CD138 enhances sensitivity to Bortezomib treatment. a Schematic of experimental design and treatment conditions. b Comparison of tumor burden in tibia, $n=5$ mice per condition. Mann-Whitney $t$ test analysis conducted. Errors reflect SD
A

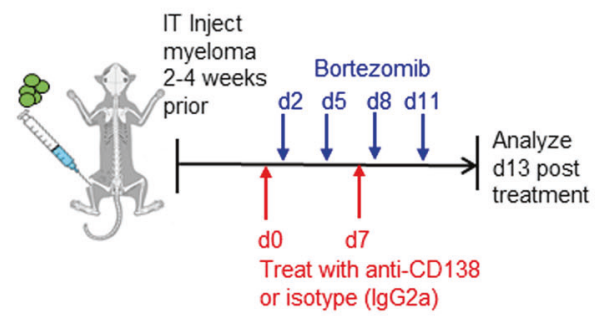

B

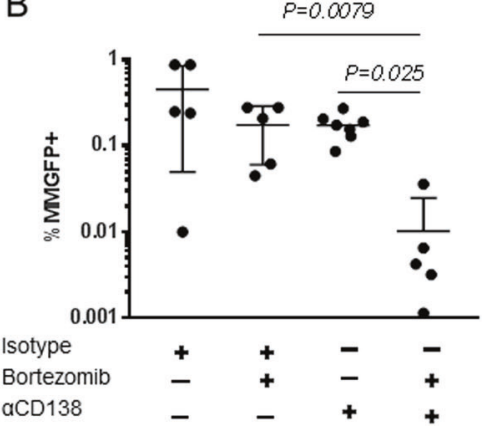

\section{Discussion}

Whereas previous studies have looked at stable populations of CD138 + and CD138 - clone [24], one of the novelties of our study has been to resolve the dynamics and interconversion of the two groups in vivo and during tumor progression. Thus, we have discovered that CD138 surface expression demarcates two distinct functional states for myeloma cells in vivo. CD138-high expressers have enhanced proliferation and survival, leading to better tumor growth in the BM. In contrast, CD138-neg expressers are highly motile, and promote dissemination and spreading throughout the body. CD138 expression is highly dynamic, controlled by extrinsic unknown factors found in serum, and changes in expression allow myeloma to be highly responsive to its microenvironment. We suspect the differences in dissemination for CD138-neg cells over CD138high cells reflect differences in BM retention occurring during initial hours following injection into the bone, and after which, growth of CD138-high and CD138-neg injected subsets proceeds similarly. Although other surface markers may also be controlled by extrinsic factors in serum and tumor size, we find that blockade of CD138 was sufficient to induce intravasation further confirming that CD138 was directly involved and not merely a biomarker of these two states.

Based on these findings we propose a model in which CD138 expression cycles to promote tumor growth and dissemination. During periods of growth, myeloma cells upregulate CD138 surface expression to bind and accumulate survival and growth factors like IL-6 to promote proliferation and survival. CD138 adhesion may inhibit migration, keeping cells in nutrient-rich environments. As tumors expand and overtake nutrient capacity in the microenvironment, CD138 surface expression rapidly internalizes via endocytosis. CD138 downregulation drives cells into a pro-dissemination state by reducing adhesion, leading to more motility in BM parenchyma and egress through blood vessels. After myeloma cells intravasate, blood serum factors can restore CD138 surface expression, which enhances re-engraftment and initiation of a new focus of growth, elsewhere.

Many factors can be involved in the regulation of the CD138 surface expression, although not in the context of myeloma dissemination. Syntenin and PIP2 have been implicated in controlling endocytic trafficking of CD138 via Arf6-labeled endosomes in epithelial cells [11]. Recycling to the surface was blocked by serum starvation, whereas internalization was not dependent on these adaptors. Analysis of myeloma samples freshly collected from multiple myeloma patients showed that CD138 levels drop over time [42], and is therefore a poor marker in the clinic. Downregulation of surface CD138 has been attributed to early stage apoptosis [30], but we find new migratory functions associated with CD138-negative multiple myeloma cells. CD138-negative myeloma cells have been reported in Xbp1-deficient myeloma cells, leading to dedifferentiation and resistance to bortezomib treatment [43]. However, we did not detect an appreciable difference in XBP1 expression in CD138 subsets in our model (data not shown), and moreover, CD138 expression was rapidly reversible, which would be inconsistent with dedifferentiation.

Myeloma spreading and migration are important drivers of the disease progression. Although myeloma cells are known to be disseminated throughout the body, multiple clones with heterogeneous mutations with different sensitivities to chemotherapies are not uniformly distributed throughout the body [44, 45]. During relapse, drugresistant clones are proliferating and spreading from bone to bone in order to disseminate and colonize the marrow. Treating patient with anti-CD138 therapies [46], which may temporarily reduce bulk tumor burden and decrease myeloma adhesion to BM stroma cells, could ultimately be detrimental by triggering CD138 downregulation, leading to more dissemination, and possibly selection of CD138-neg myeloma clones, which have been reported to be highly malignant [32]. This may explain why anti-CD138 therapy has not been successful in the clinic as a single agent. Since anti-CD138 can both enhance cell death and mobilize myeloma from BM, 
further studies will need to determine which pathways are synergizing with bortezomib treatment. Understanding how new drugs alter myeloma migration and BM retention in vivo may help predict their potential utility in patients. New therapies targeting myeloma spreading should be further explored and may synergize with current traditional chemotherapies leading to better outcomes.

Acknowledgements This work was supported by the National Institutes of Health and Einstein start-up funds. We would like to thank Jeff Segall for critical feedback, Sonia Elhadad for preliminary studies and technical assistance.

Author contributions MJM, IA, and DRF performed the experiments. DRF designed the research and wrote the paper. MC and PLB developed Vk*MYC system and provided key insights. SP provided the human samples, and key insights.

\section{Compliance with ethical standards}

Conflict of interest The authors declare that they have no conflict of interest.

Publisher's note: Springer Nature remains neutral with regard to jurisdictional claims in published maps and institutional affiliations.

\section{References}

1. Kumar SK, Dispenzieri A, Lacy MQ, Gertz MA, Buadi FK, Pandey S, et al. Continued improvement in survival in multiple myeloma: changes in early mortality and outcomes in older patients. Leukemia. 2014;28:1122-8. https://doi.org/10.1038/leu. 2013.313.

2. Boise LH, Kaufman JL, Bahlis NJ, Lonial S, Lee KP. The Tao of myeloma. Blood. 2014;124:1873-9. https://doi.org/10.1182/ blood-2014-05-578732.

3. Fairfield H, Falank C, Avery L, Reagan MR. Multiple myeloma in the marrow: pathogenesis and treatments. Ann N Y Acad Sci. 2016;1364:32-51. https://doi.org/10.1111/nyas.13038.

4. Di Marzo L, Desantis V, Solimando AG, Ruggieri S, Annese T, Nico B, et al. Microenvironment drug resistance in multiple myeloma: emerging new players. Oncotarget. 2016;7:60698-711. https://doi.org/10.18632/oncotarget.10849.

5. Roccaro AM, Sacco A, Purschke WG, Moschetta M, Buchner K, Maasch C, et al. SDF-1 inhibition targets the bone marrow niche for cancer therapy. Cell Rep. 2014;9:118-28. https://doi.org/10. 1016/j.celrep.2014.08.042.

6. Azab AK, Runnels JM, Pitsillides C, Moreau AS, Azab F, Leleu $\mathrm{X}$, et al. CXCR4 inhibitor AMD3100 disrupts the interaction of multiple myeloma cells with the bone marrow microenvironment and enhances their sensitivity to therapy. Blood. 2009;113: 4341-51. https://doi.org/10.1182/blood-2008-10-186668.

7. Rollig C, Knop S, Bornhauser M. Multiple myeloma. Lancet. 2015;385:2197-208. https://doi.org/10.1016/S0140-6736(14) 60493-1.

8. Stepp MA, Gibson HE, Gala PH, Iglesia DD, Pajoohesh-Ganji A, Pal-Ghosh S, et al. Defects in keratinocyte activation during wound healing in the syndecan-1-deficient mouse. J Cell Sci. 2002;115:4517-31.

9. Koda JE, Rapraeger A, Bernfield M. Heparan sulfate proteoglycans from mouse mammary epithelial cells. Cell surface proteoglycan as a receptor for interstitial collagens. J Biol Chem. 1985;260:8157-62.

10. Freissler E, Meyer auf der Heyde A, David G, Meyer TF, Dehio C. Syndecan-1 and syndecan-4 can mediate the invasion of OpaHSPG-expressing Neisseria gonorrhoeae into epithelial cells. Cell Microbiol. 2000;2:69-82.

11. Yao W, Rose JL, Wang W, Seth S, Jiang H, Taguchi A, et al. Syndecan 1 is a critical mediator of macropinocytosis in pancreatic cancer. Nature. 2019;568:410-4. https://doi.org/10.1038/ s41586-019-1062-1.

12. Stewart MD, Ramani VC, Sanderson RD. Shed syndecan-1 translocates to the nucleus of cells delivering growth factors and inhibiting histone acetylation: a novel mechanism of tumor-host cross-talk. J Biol Chem. 2015;290:941-9. https://doi.org/10.1074/ jbc.M114.608455.

13. Beauvais DM, Ell BJ, McWhorter AR, Rapraeger AC. Syndecan1 regulates alphavbeta 3 and alphavbeta5 integrin activation during angiogenesis and is blocked by synstatin, a novel peptide inhibitor. J Exp Med. 2009;206:691-705. https://doi.org/10.1084/jem. 20081278.

14. Rapraeger AC. Syndecan-regulated receptor signaling. J Cell Biol. 2000;149:995-8.

15. Coombe DR. Biological implications of glycosaminoglycan interactions with haemopoietic cytokines. Immunol Cell Biol. 2008;86:598-607. https://doi.org/10.1038/icb.2008.49.

16. Casu B, Naggi A, Torri G. Heparin-derived heparan sulfate mimics to modulate heparan sulfate-protein interaction in inflammation and cancer. Matrix Biol. 2010;29:442-52. https:// doi.org/10.1016/j.matbio.2010.04.003.

17. Bernfield M, Gotte M, Park PW, Reizes O, Fitzgerald ML, Lincecum J, et al. Functions of cell surface heparan sulfate proteoglycans. Annu Rev Biochem. 1999;68:729-77. https://doi.org/ 10.1146/annurev.biochem.68.1.729.

18. Hayashida K, Bartlett AH, Chen Y, Park PW. Molecular and cellular mechanisms of ectodomain shedding. Anat Rec. 2010;293:925-37. https://doi.org/10.1002/ar.20757.

19. Lonial S, Durie B, Palumbo A, San-Miguel J. Monoclonal antibodies in the treatment of multiple myeloma: current status and future perspectives. Leukemia. 2016;30:526-35. https://doi.org/ 10.1038/leu.2015.223.

20. Polson AG, Sliwkowski MX. Toward an effective targeted chemotherapy for multiple myeloma. Clinical cancer research: an official journal of the American Association for. Cancer Res. 2009;15:3906-7. https://doi.org/10.1158/1078-0432.CCR-090572.

21. Yoo EM, Trinh KR, Tran D, Vasuthasawat A, Zhang J, Hoang B, et al. Anti-CD138-targeted interferon is a potent therapeutic against multiple myeloma. J Interferon Cytokine Res. 2015;35:281-91. https://doi.org/10.1089/jir.2014.0125.

22. McCarron MJ, Park PW, Fooksman DR. CD138 mediates selection of mature plasma cells by regulating their survival. Blood. 2017;129:2749-59. https://doi.org/10.1182/blood-201701-761643.

23. Reijmers RM, Spaargaren M, Pals ST. Heparan sulfate proteoglycans in the control of B cell development and the pathogenesis of multiple myeloma. FEBS J. 2013;280:2180-93. https:// doi.org/10.1111/febs.12180.

24. Khotskaya YB, Dai Y, Ritchie JP, MacLeod V, Yang Y, Zinn K, et al. Syndecan-1 is required for robust growth, vascularization, and metastasis of myeloma tumors in vivo. $\mathrm{J}$ Biol Chem. 2009;284:26085-95. https://doi.org/10.1074/jbc.M109.018473.

25. Moreaux J, Sprynski AC, Dillon SR, Mahtouk K, Jourdan M, Ythier A, et al. APRIL and TACI interact with syndecan-1 on the surface of multiple myeloma cells to form an essential survival loop. Eur J Haematol. 2009;83:119-29. https://doi.org/10.1111/j. 1600-0609.2009.01262.x. 
26. Derksen PW, de Gorter DJ, Meijer HP, Bende RJ, van Dijk M, Lokhorst HM, et al. The hepatocyte growth factor/Met pathway controls proliferation and apoptosis in multiple myeloma. Leukemia. 2003;17:764-74. https://doi.org/10.1038/sj.leu.2402875.

27. Mahtouk K, Cremer FW, Reme T, Jourdan M, Baudard M, Moreaux J, et al. Heparan sulphate proteoglycans are essential for the myeloma cell growth activity of EGF-family ligands in multiple myeloma. Oncogene. 2006;25:7180-91. https://doi.org/10. 1038/sj.onc.1209699.

28. Beauvais DM, Jung O, Yang Y, Sanderson RD, Rapraeger AC. Syndecan-1 (CD138) Suppresses Apoptosis in Multiple Myeloma by Activating IGF1 Receptor: Prevention by SynstatinIGF1R Inhibits Tumor Growth. Cancer Res. 2016;76:4981-93. https:// doi.org/10.1158/0008-5472.CAN-16-0232.

29. Wu YH, Yang CY, Chien WL, Lin KI, Lai MZ. Removal of syndecan-1 promotes TRAIL-induced apoptosis in myeloma cells. J Immunol. 2012;188:2914-21. https://doi.org/10.4049/jimmunol. 1102065 .

30. Jourdan M, Ferlin M, Legouffe E, Horvathova M, Liautard J, Rossi JF, et al. The myeloma cell antigen syndecan-1 is lost by apoptotic myeloma cells. Br J Haematol. 1998;100:637-46.

31. Seidel C, Borset M, Hjertner O, Cao D, Abildgaard N, HjorthHansen $\mathrm{H}$, et al. High levels of soluble syndecan-1 in myelomaderived bone marrow: modulation of hepatocyte growth factor activity. Blood. 2000;96:3139-46.

32. Kawano Y, Fujiwara S, Wada N, Izaki M, Yuki H, Okuno Y, et al. Multiple myeloma cells expressing low levels of CD138 have an immature phenotype and reduced sensitivity to lenalidomide. Int $\mathbf{J}$ Oncol. 2012;41:876-84. https://doi.org/10.3892/ijo.2012.1545.

33. Ghosh N, Matsui W. Cancer stem cells in multiple myeloma. Cancer Lett. 2009;277:1-7. https://doi.org/10.1016/j.canlet.2008. 08.005 .

34. Paino T, Sarasquete ME, Paiva B, Krzeminski P, San-Segundo L, Corchete LA, et al. Phenotypic, genomic and functional characterization reveals no differences between CD138++ and CD138low subpopulations in multiple myeloma cell lines. PloS ONE. 2014;9: e92378 https://doi.org/10.1371/journal.pone.0092378.

35. Chaidos A, Barnes CP, Cowan G, May PC, Melo V, Hatjiharissi $\mathrm{E}$, et al. Clinical drug resistance linked to interconvertible phenotypic and functional states of tumor-propagating cells in multiple myeloma. Blood. 2013;121:318-28. https://doi.org/10.1182/ blood-2012-06-436220.

36. Chesi M, Robbiani DF, Sebag M, Chng WJ, Affer M, Tiedemann $\mathrm{R}$, et al. AID-dependent activation of a MYC transgene induces multiple myeloma in a conditional mouse model of post-germinal center malignancies. Cancer Cell. 2008;13:167-80. https://doi. org/10.1016/j.ccr.2008.01.007.

37. Chesi M, Matthews GM, Garbitt VM, Palmer SE, Shortt J, Lefebure $M$, et al. Drug response in a genetically engineered mouse model of multiple myeloma is predictive of clinical efficacy. Blood. 2012;120:376-85. https://doi.org/10.1182/blood2012-02-412783.

38. Chesi M, Mirza NN, Garbitt VM, Sharik ME, Dueck AC, Asmann $\mathrm{YW}$, et al. IAP antagonists induce anti-tumor immunity in multiple myeloma. Nat Med. 2016. https://doi.org/10.1038/nm.4229.

39. Jensen JL, Rakhmilevich A, Heninger E, Broman AT, Hope C, Phan F, et al. Tumoricidal effects of macrophage-activating immunotherapy in a murine model of relapsed/refractory multiple myeloma. Cancer Immunol Res. 2015;3:881-90. https://doi.org/ 10.1158/2326-6066.CIR-15-0025-T.

40. Alexander CM, Reichsman F, Hinkes MT, Lincecum J, Becker $\mathrm{KA}$, Cumberledge $\mathrm{S}$, et al. Syndecan-1 is required for Wnt-1induced mammary tumorigenesis in mice. Nat Genet. 2000;25:329-32. https://doi.org/10.1038/77108.

41. Teng YH, Aquino RS, Park PW. Molecular functions of syndecan-1 in disease. Matrix Biol: J Int Soc Format Biol. 2012;31:3-16. https://doi.org/10.1016/j.matbio.2011.10.001.

42. Frigyesi I, Adolfsson J, Ali M, Christophersen MK, Johnsson E, Turesson I, et al. Robust isolation of malignant plasma cells in multiple myeloma. Blood. 2014;123:1336-40. https://doi.org/10. 1182/blood-2013-09-529800.

43. Leung-Hagesteijn C, Erdmann N, Cheung G, Keats JJ, Stewart $\mathrm{AK}$, Reece DE, et al. Xbp1s-negative tumor B cells and preplasmablasts mediate therapeutic proteasome inhibitor resistance in multiple myeloma. Cancer Cell. 2013;24:289-304. https://doi. org/10.1016/j.ccr.2013.08.009.

44. Rasche L, Chavan SS, Stephens OW, Patel PH, Tytarenko R, Ashby $\mathrm{C}$, et al. Spatial genomic heterogeneity in multiple myeloma revealed by multi-region sequencing. Nat Commun. 2017;8:268 https://doi.org/10.1038/s41467-017-00296-y.

45. Mithraprabhu S, Khong T, Ramachandran M, Chow A, Klarica D, Mai L, et al. Circulating tumour DNA analysis demonstrates spatial mutational heterogeneity that coincides with disease relapse in myeloma. Leukemia. 2017;31:1695-705. https://doi. org/10.1038/leu.2016.366.

46. Ikeda H, Hideshima T, Fulciniti M, Lutz RJ, Yasui H, Okawa Y, et al. The monoclonal antibody nBT062 conjugated to cytotoxic Maytansinoids has selective cytotoxicity against CD138-positive multiple myeloma cells in vitro and in vivo. Clin Cancer Res. 2009;15:4028-37. https://doi.org/10.1158/1078-0432.CCR-08-2867. 